\title{
Altered Structural Connectivity in Neonates at Genetic Risk for Schizophrenia: A Combined Study Using Morphological and White Matter Networks
}

\author{
Feng Shi ${ }^{\mathrm{a}}$, Pew-Thian Yap ${ }^{\mathrm{a}}$, Wei Gao ${ }^{\mathrm{b}}$, Weili Lin ${ }^{\mathrm{b}}$, John H. Gilmore ${ }^{\mathrm{c}}$, and Dinggang Shen ${ }^{\mathrm{a},{ }^{*}}$ \\ aIDEA Lab, Department of Radiology and BRIC, University of North Carolina at Chapel Hill \\ bMRI Lab, Department of Radiology and BRIC, University of North Carolina at Chapel Hill \\ cDepartment of Psychiatry, University of North Carolina at Chapel Hill
}

\section{Abstract}

Recently, an increasing body of evidence suggests that developmental abnormalities related to schizophrenia may occur as early as the neonatal stage. Impairments of brain gray matter and wiring problems of axonal fibers are commonly suspected to be responsible for the disconnection hypothesis in schizophrenia adults, but significantly less is known in neonates. In this study, we investigated 26 neonates who were at genetic risk for schizophrenia and 26 demographically matched healthy neonates using both morphological and white matter networks to examine possible brain connectivity abnormalities. The results showed that both populations exhibited small-world network topology. Morphological network analysis indicated that the brain structural associations of the high-risk neonates tended to have globally lower efficiency, longer connection distance, and less number of hub nodes and edges with relatively higher betweenness. Subgroup analysis showed that male neonates were significantly disease-affected, while the female neonates were not. White matter network analysis, however, showed that the fiber networks were globally unaffected, although several subcortical-cortical connections had significantly less number of fibers in high-risk neonates. This study provides new evidences in support of the disconnection hypothesis, reinforcing the notion that the genetic risk of schizophrenia induces alterations in both gray matter structural associations and white matter connectivity.

\section{Keywords}

High genetic risk; newborn infant; diffusion tensor imaging; schizophrenia; brain development; network analysis

\section{Introduction}

Schizophrenia is a chronic, severe, and disabling mental disorder characterized by diverse clinical presentations, such as hallucinations, delusions, anhedonia, avolition and impaired cognitive functions (Schultz and Andreasen, 1999). It is commonly accepted that

\footnotetext{
(C) 2012 Elsevier Inc. All rights reserved.

Corresponding author: Dinggang Shen, Professor, Department of Radiology and BRIC, University of North Carolina at Chapel Hill, 130 Mason Farm Road, Chapel Hill, NC 27599-7513, dgshen@med.unc.edu, Phone: 919-966-3535, Fax: 919-843-2641.

Publisher's Disclaimer: This is a PDF file of an unedited manuscript that has been accepted for publication. As a service to our customers we are providing this early version of the manuscript. The manuscript will undergo copyediting, typesetting, and review of the resulting proof before it is published in its final citable form. Please note that during the production process errors may be discovered which could affect the content, and all legal disclaimers that apply to the journal pertain.
} 
schizophrenia is not a result of regionally isolated pathologies, but is due to the alteration of interactions between two or more regions (Friston, 1998). However, the vast majority of studies on schizophrenia involved only adult subjects, and the impact of genetic risk of the illness on infants is still poorly understood.

The symptoms of schizophrenia usually start to present at late adolescence and early adulthood, and persist for a lifetime in one percent of the population (Regier et al., 1993). Interestingly, our recent work suggested that the precursor of developmental disorder may occur as early as infancy (Gilmore et al., 2010). We found structural abnormalities, such as significantly larger intracranial, CSF, and lateral ventricular volumes, in (especially male) offspring of schizophrenic parents. No significant differences in diffusion properties, however, were reported for major white matter fiber bundles. Note that having an affected relative increases the risk for schizophrenia. For example, 20-50\% of children born to schizophrenic mothers have been found to exhibit developmental abnormalities (Marcus et al., 1993). Their risk of developing schizophrenia is about 10 times higher than that of the general population (Sullivan, 2005). High-risk neonates, with minimal exposure to environmental influences, are ideal candidates for understanding the genetic effects of schizophrenia at a very early stage.

Recent advances in graph-based network analysis techniques allows for a systems-level evaluation of brain interregional interactions (Watts and Strogatz, 1998). The brain can be modeled as a complex network composed of a collection of nodes that are connected by edges. The nodes represent the anatomical regions of interest (ROIs), and the edges encode the interaction between the nodes. For T1-/T2-weighted images, the structural associations between different GM regions can be evaluated via the correlations of morphological descriptors, such as gray matter (GM) volumes, cortical thickness, and surface areas (Bassett et al., 2008; Gong et al., 2009b; He et al., 2007). Brain morphological networks, formed by collections of such associations, have been successfully employed in many studies, such as asymmetry analysis of normal aging (Zhu et al., 2012) and brain alteration analysis in relation to multiple sclerosis (He et al., 2009). Of note, Bassett et al. constructed a morphological network on brain multimodal association areas using interregional graymatter volumes, and reported the loss of frontal and the emergence of nonfrontal hubs, and increased connection distance in schizophrenia adults (Bassett et al., 2008). Diffusionweighted imaging, on the other hand, captures local microstructural information of brain tissues via water diffusion, and allows tracing of neuronal fibers in vivo. Fiber connections between each pair of brain regions can be used to construct a so-called brain white matter network (Gong et al., 2009a; He and Evans, 2010; Sporns et al., 2005). Zalesky et al. (Zalesky et al., 2010) constructed the white matter network of schizophrenia adults and found evidences of disruption of axonal connections comprising medial frontal, parietal/ occipital, and the left temporal lobe.

The disconnection hypothesis has gathered increasing support from neurophysiological and neuroimaging studies, suggesting that the core symptoms of schizophrenia are related to aberrant connectivity between distinct brain regions. However, the actual cause of dysconnectivity in schizophrenia is still controversial. Dysconnectivity may be a result of (1) aberrant synaptic plasticity, and/or (2) the "miswiring" of axonal fibers in white matter (WM) (Friston, 1999; Stephan et al., 2006). Recent studies reveal that synaptic plasticity contributes to macro-scale volumetric changes and thus can be measured by using gray matter volumes in MR brain images (Anderson, 2011; Zatorre et al., 2012). Several structural imaging studies on adults (Glahn et al., 2008; Thompson et al., 2001) provide additional evidence in support of the occurrence of gray matter abnormalities in association with the illness. This motivates us to investigate the impairment of structural associations/ interactions in gray matter (i.e., morphological network) for exploring the disconnection 
hypothesis. On the other hand, the integrity of axonal fibers can be assessed more directly with diffusion-weighted imaging by tracing the white matter connections between brain regions. Combining morphological and white matter networks takes into account the two possible causes of disconnection hypothesis and allows us to perform a more comprehensive examination of the pathologies associated with schizophrenia.

Our aim in the current work is to identify possible neurological alterations in terms of interregional interactions in high-risk neonates. We hypothesize that the high-risk neonates may be susceptible to neurological alterations at a systems level. To test this hypothesis, we investigated both morphological and white matter brain networks in neonates who were at genetic risk for schizophrenia. Network properties were extracted, and comparisons were made between 26 high-risk neonates and 26 matched healthy neonates. Details of methods and experiments are described in the following sections.

\section{Materials and Methods}

\subsection{Participants and MR image acquisition}

This study was approved by the institutional review board of the University of North Carolina School of Medicine. New mothers with schizophrenia or schizoaffective disorder were recruited and underwent a Structured Clinical Interview for DSM-IV Axis I Disorders (SCID). Past psychiatric records were obtained and a final consensus diagnosis was assigned. The matched control subjects were selected from a companion study of normal brain development. Potential control mothers were screened for psychiatric illness by using a modified SCID. Written informed consent forms were obtained from all the parents. The presence of abnormalities on fetal ultrasound, or major medical or psychotic illness in the mother, was taken as exclusion criteria. All infants were free of congenital anomalies, metabolic disease, and focal lesions. Control infants were matched on gender, age in terms of time since last menstrual period at birth, and also age since the last menstrual period at MRI (Table 1). Note that the mothers with schizophrenia had significantly less education years (10.0, SD 3.6) than that of control mothers (14.2, SD 2.7). Also, they were more likely to take antipsychotic and antidepressant medications as well as smoke during pregnancy. Detailed recruiting information can be found in (Gilmore et al., 2010).

MR images were collected using a Siemens head-only 3T scanner. None of the neonates was sedated for MRI. Before the subjects were imaged, they were fed, swaddled, and fitted with ear protection. For each neonate, T1 images with 160 axial slices were obtained with a 3D MP-RAGE sequence using imaging parameters: TR $=1820 \mathrm{~ms}, \mathrm{TE}=4.38 \mathrm{~ms}$, Flip Angle $=7^{\circ}$, and resolution $=1 \times 1 \times 1 \mathrm{~mm}^{3}$. T2 images of 70 axial slices were obtained with a turbo spin echo sequence using parameters: $\mathrm{TR}=7380 \mathrm{~ms}, \mathrm{TE}=119 \mathrm{~ms}$, flip angle $=150^{\circ}$, and voxel resolution $=1.25 \times 1.25 \times 1.95 \mathrm{~mm}^{3}$. Diffusion-weighted images were obtained for 45 axial slices using parameters: TR $=5200 \mathrm{~ms}, \mathrm{TE}=73 \mathrm{~ms}$, and resolution $=2 \times 2 \times 2 \mathrm{~mm}^{3} .7$ images were acquired, one without diffusion gradient $(b=0)$ and the remaining six with noncollinear diffusion gradient directions $(1,0,1),(-1,0,1),(0,1,1),(0,1,-1),(1,1,0)$, and $(-1,1,0)$ at $b=1,000 \mathrm{sec} / \mathrm{mm}^{2}$. For each neonate, five sets of images (with each set as an average of two scans) were acquired to improve the signal-to-noise ratio.

As summarized in Table 1, 26 high-risk neonates (12 males and 14 females) and 26 control neonates (12 males and 14 females) were used in the morphological network construction and analysis. The gestational age of the high-risk neonates was $38.1 \pm 3.1$ weeks at birth and 43.2 \pm 3.3 weeks at MRI. The gestational age of the control neonates was $38.5 \pm 2.3$ weeks at birth and 42.6 \pm 2.4 weeks at MRI. No significant difference was found for both gender and age of the two groups. 
By removing 7 subjects with insufficient quality of diffusion tensor images, a subset of subjects, including 21 high-risk neonates ( 7 males and 14 females) and 25 control neonates (11 males and 14 females), were used in the subsequent white matter network construction and analysis. The gestational age of the high-risk neonates was $37.9 \pm 3.3$ weeks at birth and $43.1 \pm 3.6$ weeks at MRI. The gestational age of the control neonates was $38.1 \pm 2.6$ weeks at birth and $42.8 \pm 2.2$ weeks at MRI. No significant difference was found for both gender and age of the two groups.

\subsection{Overview of network construction and analysis}

Schematic diagram of the network construction and analysis procedure is shown in Fig. 1. Upon computing the inter-regional GM structural associations and WM fiber connections, we constructed morphological networks and white matter networks for both high-risk and normal neonates. Graph-theoretic network analysis was then applied for analysis of these networks to determine between group differences. Network property metrics such as clustering coefficient, path length, network efficiency, small-worldness, and centrality were computed for comparison purposes. Details on structural and diffusion image processing and network construction were provided in the following subsections.

\subsection{Structural MR image processing and morphological network construction}

All structural MR images were processed with a standard pipeline, which included resampling, skull stripping, tissue segmentation, spatial normalization, and ROI volume calculation. Specifically, images were resampled to have an isotropic $1 \times 1 \times 1 \mathrm{~mm}^{3}$ resolution and then skull-stripped. The skull stripping results were further manually edited to ensure clean skull and dura removal. Tissue segmentation was performed using a coupled-level-sets based algorithm that is dedicated to neonatal segmentation (Wang et al., 2011). The segmented images were spatially normalized (Shen and Davatzikos, 2002) to a template with 90 anatomically labeled cortical and subcortical regions (see Table T1 in the supplemental material). Note that this template was adapted from (Tzourio-Mazoyer et al., 2002) to a neonatal space (Shi et al., 2011) to avoid the subject-template registration errors resulting from substantial infant-adult anatomical variances. Using the estimated deformation fields, local brain volume changes relative to the template were computed as voxel-wise tissue density maps. Based on these maps, GM volume measurements for 90 cortical and subcortical regions were then computed for all subjects. For each ROI, linear regression was used to remove the effects of total brain volume, gender, and age from the volume measurements. The residuals resulting from the regression was used as the corrected GM volume estimates.

The across-subject volumetric correlation was computed for each pair of ROIs using the Pearson correlation and a 90×90 correlation map was thus obtained for each population (Fan et al., 2010; Zhu et al., 2012). Correlation coefficients $r$ of the correlation map were then converted to $Z$ values, which are approximately normally distributed: $Z=0.5[\ln (1+r)-\ln (1$ $-r)$ ]. The respective $z$ maps for two populations are shown in the top row of Fig. 2. By binarizing the correlation maps using a series of sparsity thresholds that will result in a certain percentages of connections, a series of unweighted and undirected graphs were obtained for subsequent network analysis.

Statistical Analysis-A non-parametric permutation test was employed to assess the statistical difference of brain network properties between two groups (He et al., 2008). First, a network property measure (e.g., efficiency, clustering, path length, or small-worldness) was computed separately for high-risk neonates and control neonates. Then, we randomly assigned the regional cortical thickness measures of each subject to either group. Correlation matrix was recomputed for each of the 2 randomized groups and a new value for the 
network property measure was then obtained. This randomization procedure was repeated 1,000 times and significance is reached if less than 5 percentile of between-group difference in the permutation distribution was greater than the observed group difference. The procedure was repeated for each sparsity threshold.

\subsection{Diffusion-weighted MR image processing and white matter network construction}

After skull-stripping, the diffusion tensors were constructed using a weighted-least-squares estimation algorithm (Zhu et al., 2007). Whole-brain streamline fiber tractography (Leemans et al., 2009) was then performed for each image with minimal seed-point FA of 0.20, minimal allowed FA of 0.10 , minimal fiber length of $20 \mathrm{~mm}$, and maximal fiber length of $200 \mathrm{~mm}$. The motivation for a low FA value allowance was to ensure that the fibers be reasonably tracked in the neonatal images, which generally have relatively lower image contrast, compared with adults. Usually, tens of thousands of fibers were tracked for each subject. The resulting fiber tracts were reviewed in ParaView (Kitware, http://www.paraview.org/), and subjects with images that were too noisy or with no diffusion-weighted MR images were excluded from the study. For each subject, the T2weighted image was rigidly aligned to the b0 image, so that the ROIs in the T2 image space could be propagated to the DTI image space. For each pair of ROIs, the number of fibers passing through the two ROIs was counted as an indication of anatomical association between the two ROIs (Gong et al., 2009a; Sporns et al., 2005; Zalesky et al., 2010), resulting in a 90 by 90 connection map for each individual subject.

Statistical Analysis-The variability of network properties in a group was assessed through the mean and standard deviation. Statistical significance of network property differences between two groups was evaluated by using the non-parametric permutation test. Besides global measurements such as efficiency, clustering, path length, or small-worldness, local connections can also be investigated. Region pairs with significant differences in the number of connecting fibers can be identified through permutation tests. Note that the statistical analysis was only performed on node pairs that had larger than average fiber connections (Zalesky et al., 2010c). The effects of age, gender, and total number of fibers were regressed out before statistical analysis. False discovery rate (FDR) with $\mathrm{p}<0.05$ was employed for multiple comparison correction.

\subsection{Graph-based network analysis}

An excellent summary of network measures can be found in (Rubinov and Sporns, 2009). We used the Brain Connectivity Toolbox (http://www.brain-connectivity-toolbox.net) to compute these network measures.

Network Sparsity-The brain network is represented by a graph $G$, composed of $N$ nodes and $L$ edges. The degree of each node $K_{I}(1 \leq i \leq N)$ is defined as the number of edges connecting the node $i$. Sparsity, also called the network cost, measures the percentage of the number of existing edges in all possible connections: $K_{\text {sparsity }}=\frac{1}{N(N-1)} \sum_{i=1}^{N} K_{i}, 0 \leq K_{\text {sparsity }}$ $\leq 1$. Thresholding based on sparsity can be performed to convert the graph into an unweighted and undirected graph, facilitating subsequent network-property analysis and comparison. A small sparsity threshold generates sparsely connected network, while a large sparsity threshold results into a densely connected network. Since there is no definitive way to select a single threshold, we thresholded the correlation matrices over a wide range of sparsity (10-25\%) and then estimated the properties of the resulting graphs at each threshold value (He et al., 2008). A fixed sparsity was however used for simplifying the investigation of the modular structure of the network. This sparsity was chosen to be the lowest value that produced a fully connected network with 90 nodes. 
Small-world Properties-Global efficiency considers global information processing and

transfer from each region to all other regions in the brain: $E_{\text {Global }}=\frac{1}{N(N-1)} \sum_{i=1, i \neq j}^{N} \frac{1}{L_{i, j}}$, where $L_{i, j}$ is the shortest absolute path length between the $i$ th node and the $j$ th node. Local efficiency of each region $i$ describes the mean transfer efficiency of the region with its neighbors, $E_{l o c}(i)=\frac{1}{N_{G_{i}}\left(N_{G_{i}}-1\right)} \sum_{j, k \in G_{i}} \frac{1}{L_{j, k}}$, where $E_{l o c}(i)$ is the local efficiency for the $\dot{t}$ th node, $G_{i}$ is a subgraph comprising nodes directly connected to node $i$, and $N_{G_{i}}$ is the node number of the subgraph $G_{i}$. By averaging over all regions, the average local efficiency for the whole brain can be obtained, $E_{\text {local }}=\frac{1}{N} \sum_{i \in G} E_{l o c}(i)$, where $G$ is the whole graph.

Clustering coefficient of a network, measuring the whole brain segregation, is the average of the absolute clustering coefficients of all nodes: $C_{p}=\frac{1}{N} \sum_{i=1}^{N} C_{i}$, where $C_{i}=\frac{E_{i}}{K_{i}\left(K_{i}-1\right) / 2}$ and $E_{i}$ is the number of edges in the subgraph $G_{i}$. The shortest absolute path length of a network, measuring the whole brain functional integration, is the average of the shortest absolute path lengths between the nodes: $L_{p}=\frac{1}{N} \sum_{i=1}^{N} L_{i}$, where $L_{i}=\frac{1}{N-1} \sum_{i=1, i \neq j}^{N} \min \left\{L_{i, j}\right\}$ and $L_{i, j}$ is the shortest absolute path length between the $i$ th node and the $j$ th node. A small-world network has similar absolute path lengths but higher absolute clustering coefficients than that of random networks with the same numbers of nodes and edges, which can be represented by the two parameters, namely normalized clustering $\gamma=C_{p}^{\text {real }} / C_{p}^{\text {rand }}>1$, and normalized path length $\lambda=L_{p}^{\text {real }} / L_{p}^{\text {rand }}$ (Watts and Strogatz, 1998). These two parameters can further be summarized as small-worldness $\sigma=\gamma / \lambda$. For reference, comparable regular lattice and random graphs were also generated over the same range of network sparsity thresholds. In particular, lattice network is constructed by composing a ring network with the same number of edges, i.e., same network sparsity, according to the real network. Random network is obtained by iteratively rewiring a pair of random edges of the existing brain network. Random network is obtained by iteratively rewiring a pair of random edges of the existing brain network. For example, two existing edges $\left(i_{1}, j_{1}\right)$ and $\left(i_{2}, j_{2}\right)$ are randomly selected and rewired into $\left(i_{1}, j_{2}\right)$ and $\left(i_{2}, j_{1}\right)$ if both of the new edges do not already exist. Random permutation is repeated until the topological structure of resulting graph is randomized. 1000 random networks are constructed and the results are averaged (Liu et al., 2008).

Connection distance-The connection distance of each edge was estimated by the Euclidean distance between the centroids of the connected regions in standard neonatal stereotactic space (Bassett et al., 2008). Defined as the average connection distance (in millimeter) over all edges in a network, the mean connection distance, $d$, captures the spatial characteristics of the network.

Modularity and centrality-A network is usually composed of several modules, e.g., community structures, which is more densely connected inside the modules than in-between the modules. A module is a subset of nodes and the modularity of a network is defined as:

$Q(m)=\sum_{s=1}^{n_{m}}\left[\frac{h_{s}}{L}-\left(\frac{T_{s}}{2 L}\right)^{2}\right]$, where $m$ is the configuration of modular organization with $n_{m}$ modules; $L$ is the total number of edges of this graph; in module $s, h_{S}$ is the total number of edges, and $T_{S}$ is the sum of the degrees of the nodes. Similarly, the modularity can be evaluated as a function of network sparsity. 
In network, the nodes highly connected to others and traversed by many paths are named hub nodes, which play an essential role for controlling the information flow in the entire network (Achard et al., 2006; He et al., 2007). Betweenness centrality is defined as the fraction of the shortest paths between any pairs of nodes that travel through the node

(Freeman, 1977): $B_{c}(i)=\sum_{j \neq k \neq i \in G} \frac{\sigma_{j, k}(i)}{\sigma_{j, k}}$, where $\sigma_{j, k}$ is the number of the shortest paths from node $j$ to $k$, and $\sigma_{j, k}(i)$ is the number of these paths that pass through node $i$. Nodes with the node betweenness values larger than the mean plus standard deviation are classified as hub nodes, i.e., $B_{c}(i)>$ mean $\left(B_{c}\right)+s t d\left(B_{c}\right)$; others are classified as non-hubs (Gong et al., 2009a). Similarly, by extending the notion of betweenness centrality from nodes to edges, hub edges are those with edge betweenness larger than the mean plus standard deviation betweenness.

\section{Results}

\subsection{Morphological network properties}

Small-world network organization-For each subject, a 90 by 90 correlation matrix was computed via the Pearson correlation of the corrected gray-matter volumes between all ROI pairs (top row of Fig. 2). The absolute values of the correlations were taken as indicators of connections (He et al., 2008). As shown in Fig. 3, both high-risk neonates and control neonates demonstrate small-world network properties, such as intermediate network efficiency in between degree-matched random and lattice networks (Fig. 3A-B), and considerably larger clustering coefficients and path lengths than those of degree-matched random networks (Fig. 3C-E). Statistical analysis indicates that high-risk neonates have significantly lower global efficiency, and higher local efficiency, clustering coefficient, and patch length. Further investigation indicates that the mean connection distance is significantly higher $(36.1 \mathrm{~mm}$ at sparsity $12 \%$ ) in high-risk neonates than control neonates (34.7 $\mathrm{mm}$ at sparsity $12 \%$ ) (See Fig. S1 in supplementary material).

Subgroup analysis for gender effects-In adults, males were reported to be more likely affected by schizophrenia, with more severe symptoms (Seeman, 1986). To investigate the gender effects in our population, we subdivided both neonatal groups into males and females and analyzed them separately, with results shown in Fig. 4. The male high-risk neonates demonstrates significantly reduced global efficiency and increased local efficiency. Female high-risk neonates are less affected and surprisingly show even higher global efficiency and lower local efficiency than those of female control neonates. Our results also suggest that male neonates are more susceptible to the effects of the genetic risk.

Modularity analysis-Brain network is composed of multiple modules working together as a whole, governed by functional specialization and integration (Rubinov and Sporns, 2009). Such topology usually has more connections within modules than between modules. A fixed sparsity of 0.12 was used, at which networks of both population reach fully connection.

In Fig. 5, the spring-embedding visualization of networks shown in the left panel was implemented with Kamada-Kawai layout algorithm using the Pajek (Batagelj and Mrvar, 1998) software package (pajek.imfm.si/doku.php). The nodes and intra-modular connections are colored-coded based on the communities detected by the algorithm described in (Clauset et al., 2004); and inter-modular connections are color-coded with light-gray. The sizes of the vertices are weighted by the logarithmically-scaled node betweenness (Freeman, 1977). The right panel shows a circular view of the network by arranging the 90 ROIs clockwise in a circle based on their indices. From the top left panel of Fig. 5, we can observe that the 
network of high-risk neonates is roughly composed of three modules. The left module is composed mainly of the frontal and temporal nodes, with the right module composed of the motor and occipital areas, and the middle module composed of the orbitofrontal and temporal regions. The information transfer between the left and right modules is mediated by the middle module, causing lower information transfer efficiency between the two modules. The middle and left modules in high-risk neonates are more integrated in the control neonates. More inter-module connections (gray edges) can be observed in the control neonates, indicating better connections for the two major modules.

Network hubs are the nodes with significantly more connections to other nodes, and hub edges are the edges that are traversed by a relatively significant number of the shortest paths connecting a pair of nodes. Hubs are important in a brain network and have direct implications on its resilience to random and targeted attacks (Achard et al., 2006; He et al., 2007). We identified the hub nodes and edges in all networks by using the betweenness centrality measure as described in the method section. In Fig. 6, hub nodes are represented by spheres, color-coded based on the modules they belong to. Hub edges are shown as lines connecting a pair of brain regions, with the thickness proportional to the traffic load of information transfer and processing. Note that the two ends of the hub edges are not necessarily hub nodes. We also list the hub nodes and the top 5 weighted edges in Tables 2 and 3. Both neonatal populations have hub nodes in frontal and temporal lobes. High-risk neonates have additional hub nodes in the occipital lobe, while the control neonates in the parietal and subcortical regions (Table 2). Moreover, high-risk neonates have less number (12) but higher average betweenness $\left(4.4 \times 10^{2}\right)$ of hub nodes than that of control neonates with number $(18)$ and betweenness $\left(3.5 \times 10^{2}\right)$, indicating that the network organization in high-risk neonates prefer huge hubs that are more vulnerable to targeted attacks (Newman, 2006). We also classified the anatomical regions as Primary, Associations, Paralimibic, and Subcortical as described by (Achard et al., 2006). Hub nodes in high-risk neonates are mostly located in the association and paralimbic cortex, while control neonates have additional hubs in the primary and subcortical cortex.

Hub edges in high-risk neonates are mostly located at the intra-frontal, frontal-temporal, occipital, and temporal-limbic regions (Table 3). This indicates that high-risk neonates have heavy traffic on the long distance connections to communicate spatially dispersed modules for functional integration, resulting in less economic information flow. And possible damages of the hub edges may remarkably affect the stability and efficiency of the network (Achard et al., 2006).

Modular analysis indicates that a similar pattern is manifested in the subgroups (Fig. S2 in supplementary material). High-risk subgroups generally have the dominating hub edges with very large betweenness in contrast to other hub edges that have much smaller betweenness. This observation is similar to the findings obtained from the whole high-risk group. However, hub edges of the control neonates have a more balanced betweenness distribution, and the number of hub edges is also higher with low average betweenness, indicating possibly better support of brain connectivity and integration. The female high-risk subgroup has a more similar pattern with the control subgroup than that of the male high-risk subgroup, suggesting that the male neonates are more susceptible to the genetic risk.

\subsection{White matter network properties}

Fiber tractography-White matter fibers were tracked for whole brain and checked manually to ensure anatomical correctness (see Fig. S3 in supplemental material for representative fiber tracking results). Connectivity matrices were constructed for all subjects based on the number of fiber connections between ROI pairs. The average connectivity matrices for two populations are shown in the bottom row of Fig. 2. 
Small-world network organization-Figs 7(A-B) show the global efficiency and local efficiency of high-risk and control neonates as functions of network sparsity. No significant difference was found in global and local efficiencies between the two neonatal populations. Mean connection distances were also non-significant (around $20.0 \mathrm{~mm}$ at sparsity $14 \%$ ) as shown in Fig. S1 in supplementary material. Neonates exhibit larger clustering coefficients and comparable path length compared with the degree-matched random networks, while no difference can be found between the high-risk neonates and control neonates (Fig. 7C-E). This finding confirms that the small-world topologies are preserved in the white matter networks of both populations.

Subgroup analysis for gender effects-Gender effects were also investigated by comparing the global and local efficiency for white matter networks (1) between male highrisk neonates and male control neonates, and (2) between female high-risk neonates and female control neonates. Few differences were found between each of two comparisons in the small-world regime and none of them survived the multiple comparison correction (FDR) for the 16 tests (each test for each sparsity from 10\% to 25\%). Results are shown in Fig. S4 of supplemental material. These results indicate that gender does not influence differently on the major white matter network metrics of both high-risk and control neonates.

Modularity analysis-A fixed sparsity of 0.14 was used, at which the two networks were fully connected. Results are shown in Fig. 8. The hub nodes can be found mostly in the subcortical regions and the hub edges mainly connect the left and right brain. This may be because the network is constructed based on fiber connections, and fiber length is generally high for inter-hemisphere connections. The two groups have similar intra-connections in subcortical regions. Control neonates have a more symmetric hub edge distribution with additional hub edges between left and right hippocampus. High-risk neonates have a leftsubcortical-cortical hub edge connecting between left putamen (PUT-L) and right middle cingulate gyrus (MCG-R). This pattern is also observed in subgroup modular analysis (Fig. S5 in supplementary material).

Impaired local fiber connections-Although there was no significant global difference, we found alterations of local fiber connections between the two populations. Of the total $(90 \times 89) / 2=4005$ interregional connections, 6 connections were found to exhibit significantly lower number of fibers in the high-risk neonates than the control neonates ( $<<0.05$, FDR corrected). As shown in Fig. 9, 4 out of 6 connections are between the right subcortical regions and cortical regions such as paracentral gyrus, temporal lobe, and occipital lobe. And, the other 2 connections are between the occipital lobe and limbic lobe. None of connections have significantly higher number of fibers in the high-risk neonates.

\section{Discussion}

In this study, we investigated brain organization in terms of morphological and white matter networks of the neonates at genetic risk for schizophrenia. These networks may reflect different causes of schizophrenia in support of the disconnection hypotheses in association with GM and WM. Both morphological and white matter networks demonstrate small-world topologies. Results indicate that the high-risk neonates exhibit significantly altered structural associations, such as reduced global efficiency, longer connection distance, and less hub nodes and edges. White matter networks, however, were globally unaffected, although several subcortical-cortical connections showed early sign of impairment with significantly lower number of fibers. The findings verify our hypothesis that the morphological and white matter networks of neonates are influenced by the high-risk but in varied degrees. Note that having a first-degree relative with schizophrenia may increase the risk to 10 times higher 
than that of a normal population (Sullivan, 2005). Thus it is expected that about 10 percent of high-risk neonates in this study may develop schizophrenia later in their lives. The observed abnormalities may represent the intermediate phenotypes of the risk.

\subsection{Altered morphological network in high-risk neonates}

The GM density on MRI is a macroscopic and indirect measure of the complex brain architecture of glia, vasculature, and neurons with dendritic and synaptic processes. Brain regions related with a certain disrupted function may cause synchronous impairments in gray matter, which can manifest as altered structural association in the morphological network. GM structural association has been found to reflect neurological dysfunction (Dazzan et al., 2004) and correlate with the functional connectivity (Bhojraj et al., 2010).

This study is the first to examine high-risk schizophrenia neonates for new evidences that altered structural associations exist as early as in the neonatal stage. Specifically, high-risk neonates show significantly reduced global efficiency, longer path length and connection distance, suggesting lower communication, which is consistent with studies on schizophrenia adults (Bassett et al., 2008). High-risk neonates also show increased local efficiency and clustering coefficient, which can be explained by the findings that brain is more modulated than that of control neonates.

Subgroup analysis shows that male subjects have a higher susceptibility to the genetic risk, which is in agreement with a volumetric analysis (Gilmore et al., 2010) where male highrisk schizophrenia neonates were found to have significantly larger intracranial, CSF, and lateral ventricular volumes. Also males were reported to have higher incidence rate (1.4:1) than females for schizophrenia (McGrath et al., 2008).

\footnotetext{
Aberrant modular structures in morphological network of high-risk neonates

-One of the most important features of complex systems, such as the large-scale brain networks, is that they generally have a modular structure (Bullmore and Sporns, 2009). Detecting modules in a network can help identify groups of brain nodes that perform specific biological functions. Results from morphological network analysis (Fig. 5) shows that the control neonates are generally better organized as functionally oriented modules, and the pattern is consistent with the modules found in young healthy adults (Chen et al., 2011). However, some regions were separated into different modules in the high-risk neonates, i.e., regions associated with brain executive, sensorimotor, decision-making, and other basic cognitive functions, which may affect their information processing efficiency and further brain communication development.
}

\subsection{Globally unaffected but locally impaired white matter network in high-risk neonates}

Although many studies suggest the white matter connectional architecture may be relatively preserved in schizophrenia patients (Stephan et al., 2006), most investigations were not based on the axonal fiber connectivity. Recently, schizophrenia adults were suggested having disrupted axonal fiber connectivity (van den Heuvel et al., 2010; Zalesky et al., 2010). The impaired connectivity involve a distributed network of nodes comprising medial frontal, parietal, occipital, and left temporal lobes. Note that the brains of high-risk neonates are under rapid development in both structures and functions and hence patterns of abnormalities might not resemble exactly as those of adults. In the present study, we employed a similar white matter network analysis and found that high-risk neonates showed globally unaffected small-world topologies. Males and females also showed no significant difference in their network organization. However, modular analysis found that high-risk neonates had asymmetric hub distributions and left-subcortical-cortical hub edges. Local fiber connection analysis indicates that the high-risk neonates suffered from impaired right- 
subcortical-cortical fiber connections, which in turn increases the information processing and transfer load for left-subcortical-cortical connections. The impaired connections involved some basic brain functions, such as motor, emotion, and vision (Andreasen, 1997; Andreasen et al., 1998). This finding is also in agreement with the results in morphological network analysis, where the high-risk neonates have less hub nodes in the subcortical regions. In general, although the impaired connections are limited in number and do not affect the whole brain topology, these results suggest that fiber connectivity alteration already exists as early as the neonatal stage and may have critical impact on subsequent brain development.

\subsection{Relationships between brain networks constructed from different modalities}

Brain networks constructed from different modalities may reflect different aspects of the underlying brain architecture (see the review (He and Evans, 2010)). The morphological and white matter networks employed in this study (also called structural networks as opposed to functional networks) are related to brain interactions as characterized by gray matter and white matter. A recent work demonstrates that there are considerable differences between the morphological network constructed with cortical thickness correlations and the white matter network constructed using DTI (Gong et al., 2012). In this study, the discrepancy in the results between morphological and white matter networks may be due to the possibility that different brain tissues are affected in a different manner or at different stage by the genetic risk factor. Information gained from different modalities may help us better understand the pathophysiology of schizophrenia.

\subsection{Limitations}

This study has several limitations. First, high-risk infants were more likely to be exposed to medications such as antipsychotic, antidepressants, and cigarette smoking during pregnancy. Previous studies have shown that the use of antipsychotics during pregnancy do not appear to significantly increase the risk of birth defects or other adverse outcomes (Einarson and Boskovic, 2009). Volumetric studies also do not find significant brain structure differences between the high-risk neonates exposed to maternal smoking and those who were not exposed (Gilmore et al., 2010). Based on this, we expect that the use of medicine and cigarette smoking has only minor effects on the results of this paper. Second, the diffusionweighted images of neonates are usually noisier than these of adults, rendering the tracked fibers less reliable. For this study, five sets of images (with each set as an average of two scans) were acquired and averaged to improve the signal-to-noise ratio. The fiber tracking results in this study was also manually reviewed and data with excessive noise were discarded. Third, the number of connecting fibers was used to define the white matter connectivity in this paper. Other measures, such as connection density, which corrects the size of end-nodes, could be further investigated (Hagmann et al., 2008). Fourth, sample size in this study is limited due to recruitment difficulties of neonates with high-genetic-risk. A larger sample size is expected in the future. Lastly, comparisons on hubs and modularity were currently made qualitatively on group-level networks. Further study may be required for quantitative evaluation of the stability of hubs and modules using sampling methods.

\subsection{Conclusion}

To our knowledge, this is the first study to investigate the brain network organization in neonates who are at high-risk for schizophrenia. We employed both morphological and white matter networks for identifying possible alterations of brain connectivity as early as in the neonatal stage. For high-risk neonates and control neonates, both networks exhibit smallworld topologies. High-risk neonates show impaired global efficiency, longer shortest path length, and longer connection distance, suggesting reduced capacity of global information integration. Subsequent modularity analysis further reveals that high-risk neonates have less 
inter-module connections, more intra-module connections, and less efficient frontal-occipital connection. Aberrant modular structures may affect the further development of brain executive, sensorimotor, decision-making, and other basic cognitive functions. White matter network shows no global network difference between high-risk and control neonates. However, several impaired subcortical-cortical connections were found, mainly those associating the basic brain functions. In general, this study provides evidences of the existence of brain alteration in neonates at genetic risk for schizophrenia, with varied degrees of alteration in structural associations and fiber connections. Future work will be focused on characterizing the dynamic network evolution of the infant brain using a longitudinal design.

\section{Supplementary Material}

Refer to Web version on PubMed Central for supplementary material.

\section{Acknowledgments}

This work was supported in part by NIH grants EB006733, EB008760, EB008374, EB009634, MH088520, MH070890, MH064065, NS055754, and HD053000.

\section{References}

Achard S, Salvador R, Whitcher B, Suckling J, Bullmore E. A resilient, low-frequency, small-world human brain functional network with highly connected association cortical hubs. The Journal of Neuroscience. 2006; 26:63-72. [PubMed: 16399673]

Anderson BJ. Plasticity of gray matter volume: The cellular and synaptic plasticity that underlies volumetric change. Developmental Psychobiology. 2011; 53:456-465. [PubMed: 21678393]

Andreasen NC. The role of the thalamus in schizophrenia. Can J Psychiatry. 1997; 42:27-33. [PubMed: 9040920]

Andreasen NC, Paradiso S, O'Leary DS. "Cognitive dysmetria" as an integrative theory of schizophrenia: a dysfunction in cortical-subcortical-cerebellar circuitry? Schizophr Bull. 1998; 24:203-218. [PubMed: 9613621]

Bassett D, Bullmore E, Verchinski B, Mattay V, Weinberger D, Meyer-Lindenberg A. Hierarchical organization of human cortical networks in health and schizophrenia. Journal of Neuroscience. 2008; 28:9239-9248. [PubMed: 18784304]

Batagelj V, Mrvar A. Pajek-program for large network analysis. Connections. 1998; 21:47-57.

Bhojraj T, Prasad K, Eack S, Francis A, Montrose D, Keshavan M. Do inter-regional gray-matter volumetric correlations reflect altered functional connectivity in high-risk offspring of schizophrenia patients? Schizophr Res. 2010; 118:62-68. [PubMed: 20171847]

Bullmore E, Sporns O. Complex brain networks: graph theoretical analysis of structural and functional systems. Nat Rev Neurosci. 2009; 10:186-198. [PubMed: 19190637]

Chen ZJ, He Y, Rosa-Neto P, Gong G, Evans AC. Age-related alterations in the modular organization of structural cortical network by using cortical thickness from MRI. NeuroImage. 2011; 56:235245. [PubMed: 21238595]

Clauset A, Newman M, Moore C. Finding community structure in very large networks. Physical Review E. 2004; 70:66111.

Dazzan P, Morgan K, Orr K, Hutchinson G, Chitnis X, Suckling J, Fearon P, Salvo J, McGuire P, Mallett R. The structural brain correlates of neurological soft signs in AESOP first-episode psychoses study. Brain. 2004; 127:143-153. [PubMed: 14570821]

Einarson A, Boskovic R. Use and safety of antipsychotic drugs during pregnancy. Journal of psychiatric practice. 2009; 15:183. [PubMed: 19461391]

Fan Y, Shi F, Smith J, Lin W, Gilmore J, Shen D. Brain anatomical networks in early human brain development. NeuroImage. 2010; 54:1862-1871. [PubMed: 20650319]

Freeman L. A set of measures of centrality based on betweenness. Sociometry. 1977; 40:35-41. 
Friston K. The disconnection hypothesis. Schizophr Res. 1998; 30:115-125. [PubMed: 9549774]

Friston KJ. Schizophrenia and the disconnection hypothesis. Acta Psychiatrica Scandinavica. 1999; 99:68-79. [PubMed: 10066009]

Gilmore JH, Kang C, Evans DD, Wolfe HM, Smith MD, Lieberman JA, Lin W, Hamer RM, Styner M, Gerig G. Prenatal and Neonatal Brain Structure and White Matter Maturation in Children at High Risk for Schizophrenia. Am J Psychiatry. 2010; 167:1083-1091. [PubMed: 20516153]

Glahn DC, Laird AR, Ellison-Wright I, Thelen SM, Robinson JL, Lancaster JL, Bullmore E, Fox PT. Meta-analysis of gray matter anomalies in schizophrenia: application of anatomic likelihood estimation and network analysis. Biological psychiatry. 2008; 64:774-781. [PubMed: 18486104]

Gong G, He Y, Chen ZJ, Evans AC. Convergence and divergence of thickness correlations with diffusion connections across the human cerebral cortex. NeuroImage. 2012; 59:1239-1248. [PubMed: 21884805]

Gong G, He Y, Concha L, Lebel C, Gross DW, Evans AC, Beaulieu C. Mapping anatomical connectivity patterns of human cerebral cortex using in vivo diffusion tensor imaging tractography. Cerebral Cortex. 2009a; 19:524. [PubMed: 18567609]

Gong G, Rosa-Neto P, Carbonell F, Chen ZJ, He Y, Evans AC. Age-and gender-related differences in the cortical anatomical network. Journal of Neuroscience. 2009b; 29:15684. [PubMed: 20016083]

Hagmann P, Cammoun L, Gigandet X, Meuli R, Honey CJ, Wedeen VJ, Sporns O. Mapping the structural core of human cerebral cortex. PLoS Biol. 2008; 6:e159. [PubMed: 18597554]

He Y, Chen Z, Evans A. Structural insights into aberrant topological patterns of large-scale cortical networks in Alzheimer's disease. The Journal of Neuroscience. 2008; 28:4756-4766. [PubMed: 18448652]

He Y, Chen ZJ, Evans AC. Small-world anatomical networks in the human brain revealed by cortical thickness from MRI. Cereb Cortex. 2007; 17:2407-2419. [PubMed: 17204824]

He Y, Dagher A, Chen Z, Charil A, Zijdenbos A, Worsley K, Evans A. Impaired small-world efficiency in structural cortical networks in multiple sclerosis associated with white matter lesion load. Brain. 2009; 132:3366-3379. [PubMed: 19439423]

He Y, Evans A. Graph theoretical modeling of brain connectivity. Current opinion in neurology. 2010; 23:341-350. [PubMed: 20581686]

Leemans, A.; Jeurissen, B.; Sijbers, J.; Jones, D. ExploreDTI: a graphical toolbox for processing, analyzing, and visualizing diffusion MR data. Annual Meeting of Intl Soc Mag Reson Med (ISMRM); 2009. p. 3537

Liu Y, Liang M, Zhou Y, He Y, Hao Y, Song M, Yu C, Liu H, Liu Z, Jiang T. Disrupted small-world networks in schizophrenia. Brain. 2008; 131:945. [PubMed: 18299296]

Marcus J, Hans SL, Auerbach JG, Auerbach AG. Children at risk for schizophrenia: The Jerusalem Infant Development Study: II. Neurobehavioral deficits at school age. Arch Gen Psychiatry. 1993; 50:797-809. [PubMed: 7692835]

McGrath J, Saha S, Chant D, Welham J. Schizophrenia: a concise overview of incidence, prevalence, and mortality. Epidemiologic reviews. 2008; 30:67-76. [PubMed: 18480098]

Newman MEJ. Modularity and community structure in networks. Proceedings of the National Academy of Sciences. 2006; 103:8577.

Regier D, Narrow W, Rae D, Manderscheid R, Locke B, Goodwin F. The de facto US mental and addictive disorders service system: Epidemiologic Catchment Area prospective 1-year prevalence rates of disorders and services. Archives of General Psychiatry. 1993; 50:85-94. [PubMed: 8427558]

Rubinov M, Sporns O. Complex network measures of brain connectivity: uses and interpretations. NeuroImage. 2009; 52:1059-1069. [PubMed: 19819337]

Schultz SK, Andreasen NC. Schizophrenia. Lancet. 1999; 353:1425-1430. [PubMed: 10227239]

Seeman MV. Current outcome in schizophrenia: women vs men. Acta Psychiatr Scand. 1986; 73:609617. [PubMed: 2875610]

Shen D, Davatzikos C. HAMMER: hierarchical attribute matching mechanism for elastic registration. IEEE transactions on medical imaging. 2002; 21:1421-1439. [PubMed: 12575879] 
Shi F, Yap PT, Wu G, Jia H, Gilmore JH, Lin W, Shen D. Infant Brain Atlases from Neonates to 1and 2-Year-Olds. PLoS ONE. 2011; 6:e18746. [PubMed: 21533194]

Sporns O, Tononi G, Kotter R. The human connectome: a structural description of the human brain. PLoS Comput Biol. 2005; 1:e42. [PubMed: 16201007]

Stephan K, Baldeweg T, Friston K. Synaptic plasticity and dysconnection in schizophrenia. Biological psychiatry. 2006; 59:929-939. [PubMed: 16427028]

Sullivan PF. The genetics of schizophrenia. PLoS medicine. 2005; 2:e212. [PubMed: 16033310]

Thompson PM, Vidal C, Giedd JN, Gochman P, Blumenthal J, Nicolson R, Toga AW, Rapoport JL. Mapping adolescent brain change reveals dynamic wave of accelerated gray matter loss in very early-onset schizophrenia. Proc Natl Acad Sci U S A. 2001; 98:11650-11655. [PubMed: 11573002]

Tzourio-Mazoyer N, Landeau B, Papathanassiou D, Crivello F, Etard O, Delcroix N, Mazoyer B, Joliot M. Automated anatomical labeling of activations in SPM using a macroscopic anatomical parcellation of the MNI MRI single-subject brain. NeuroImage. 2002; 15:273-289. [PubMed: 11771995]

van den Heuvel MP, Mandl RCW, Stam CJ, Kahn RS, Hulshoff Pol HE. Aberrant frontal and temporal complex network structure in schizophrenia: a graph theoretical analysis. The Journal of Neuroscience. 2010; 30:15915-15926. [PubMed: 21106830]

Wang L, Shi F, Lin W, Gilmore JH, Shen D. Automatic Segmentation of Neonatal Images Using Convex Optimization and Coupled Level Sets. NeuroImage. 2011; 58:805-817. [PubMed: 21763443]

Watts D, Strogatz S. Collective dynamics of 'small-world'networks. Nature. 1998; 393:440-442. [PubMed: 9623998]

Zalesky A, Fornito A, Seal M, Cocchi L, Westin C, Bullmore E, Egan G, Pantelis C. Disrupted Axonal Fiber Connectivity in Schizophrenia. Biological psychiatry. 2010; 69:80-89. [PubMed: 21035793]

Zatorre RJ, Fields RD, Johansen-Berg H. Plasticity in gray and white: neuroimaging changes in brain structure during learning. Nature neuroscience. 2012; 15:528-536.

Zhu H, Zhang H, Ibrahim J, Peterson B. Statistical analysis of diffusion tensors in diffusion-weighted magnetic resonance imaging data. Journal of the American Statistical Association. 2007; 102:1085-1102.

Zhu W, Wen W, He Y, Xia A, Anstey K, Sachdev P. Changing topological patterns in normal aging using large-scale structural networks. Neurobiology of Aging. 2012; 33:899-913. [PubMed: 20724031] 


\section{Research Highlights}

- We found that structural associations of the high-risk neonates are globally less efficiently integrated.

- Males were significantly disease-affected in morphological network, while the females were not.

- Several subcortical-cortical fiber connections were impaired. 


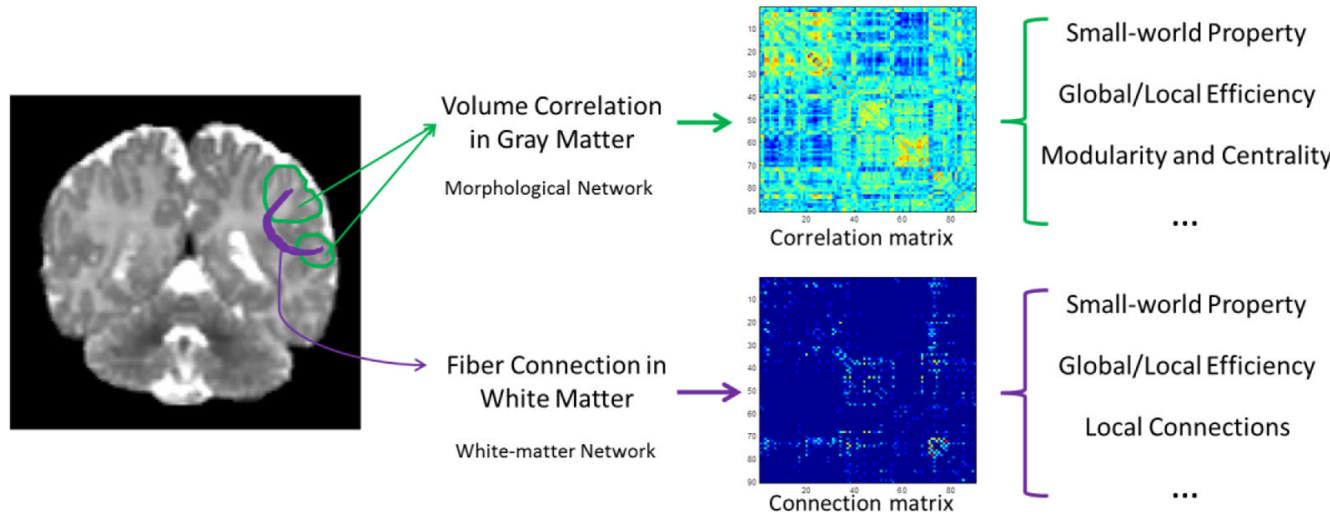

Figure 1.

Conceptual diagram illustrating the construction of the cortical morphological network using gray-matter volumes and the white matter tractography. 


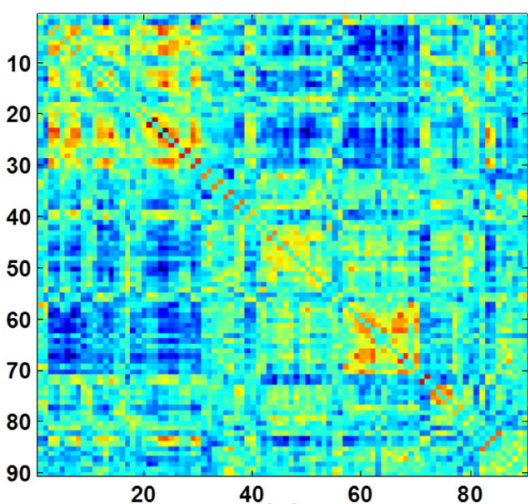

(a)

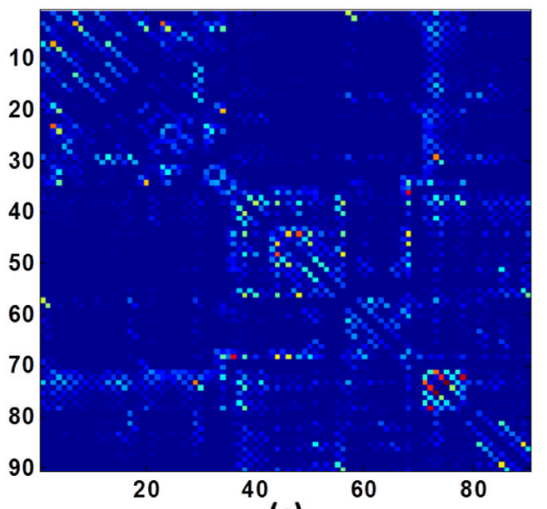

(c)

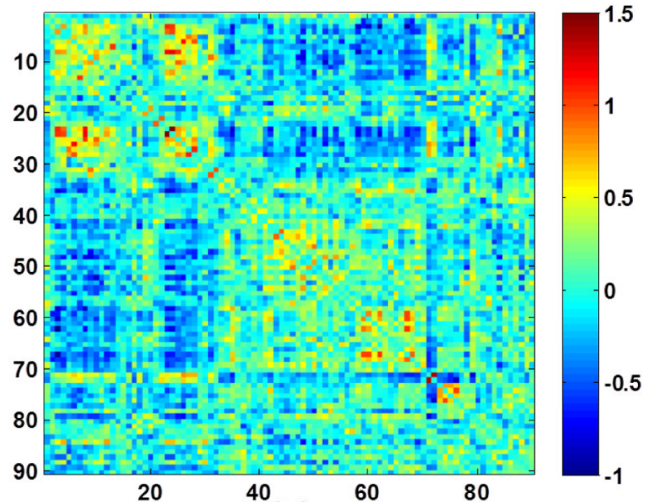

(b)

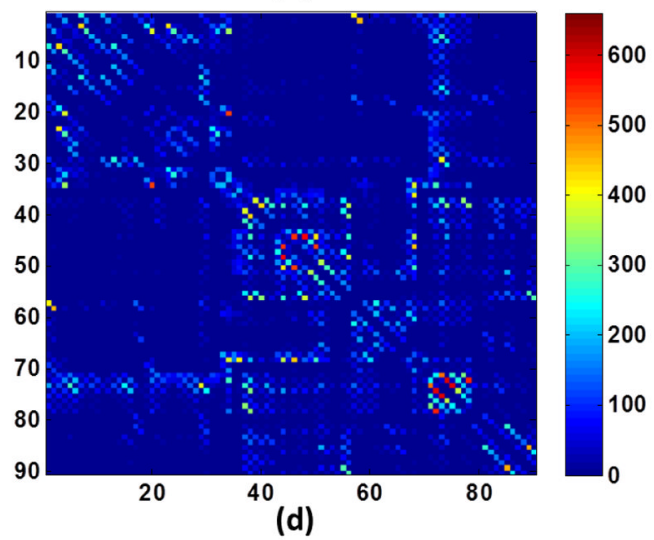

Figure 2.

Illustration of correlation matrices of morphological networks for (a) high-risk neonates and (b) control neonates, and connection matrices of white matter networks for (c) high-risk neonates and (d) control neonates. (a-b) were obtained from all subjects, respectively. (c-d) were obtained by averaging matrices from all individuals, respectively. 

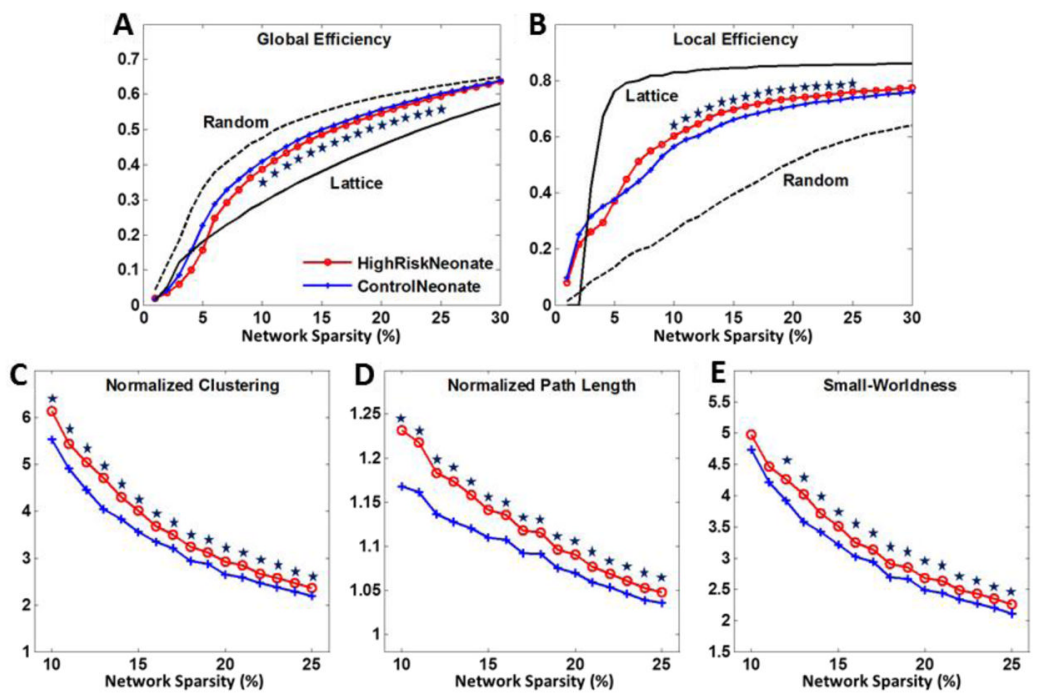

Figure 3.

Network properties of (A) global efficiency, (B) local efficiency, (C) normalized clustering, (D) normalized path length, and (E) small-worldness as functions of network sparsity for morphological networks of high-risk neonates (red) and control neonates (blue). Mean and standard deviation of network properties were illustrated at each sparsity level. Data points marked with stars indicate significant differences ( $p<0.01$ by permutation test) were achieved within sparsity of $10-25 \%$. 

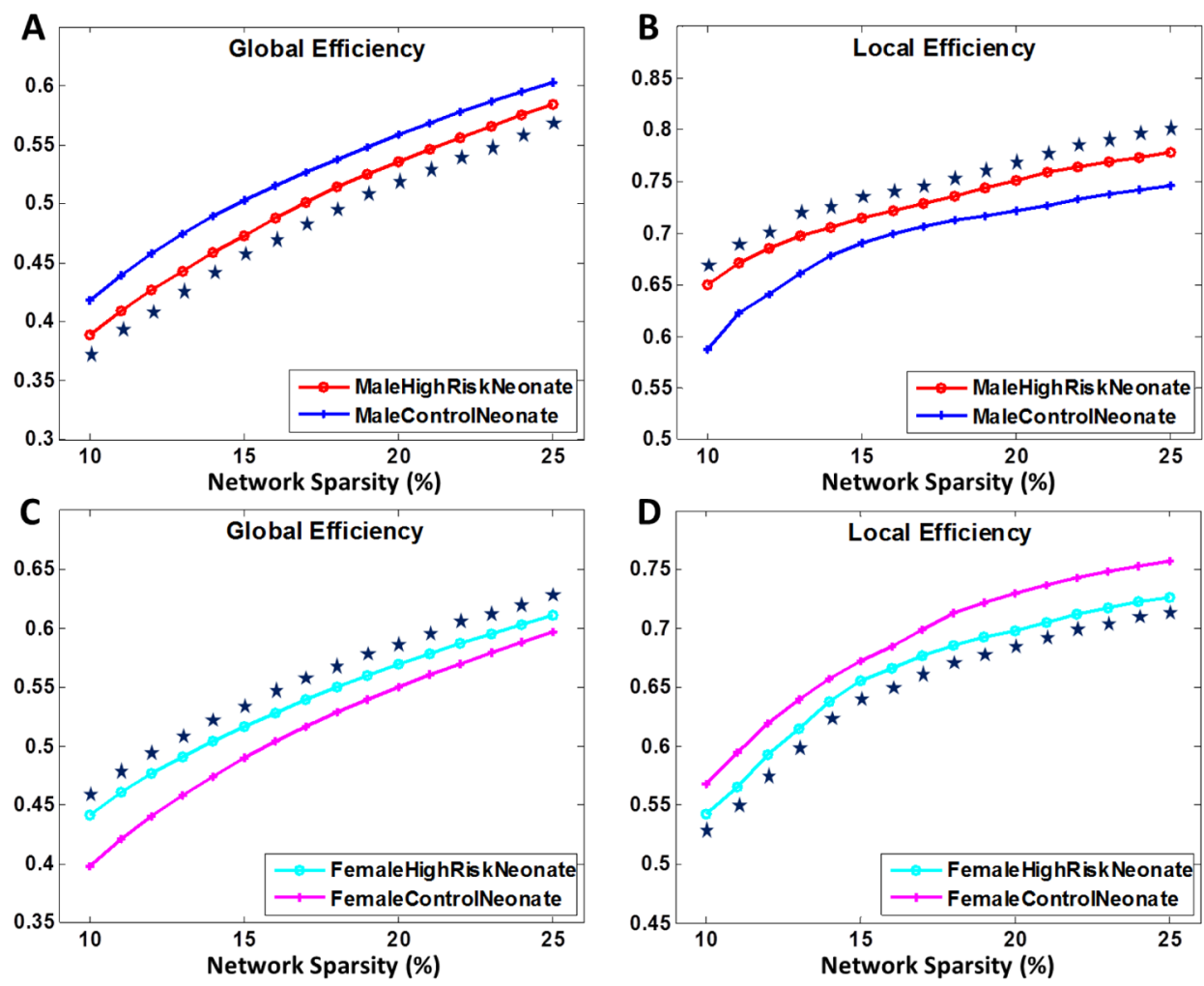

Figure 4.

Global and local efficiency for morphological networks of (A-B) male and (C-D) female high-risk neonates and control neonates as functions of network sparsity. Mean and standard deviation of network properties are illustrated at each sparsity level. Data points marked with stars indicate significant differences $(\mathrm{p}<0.01$ by permutation test). 

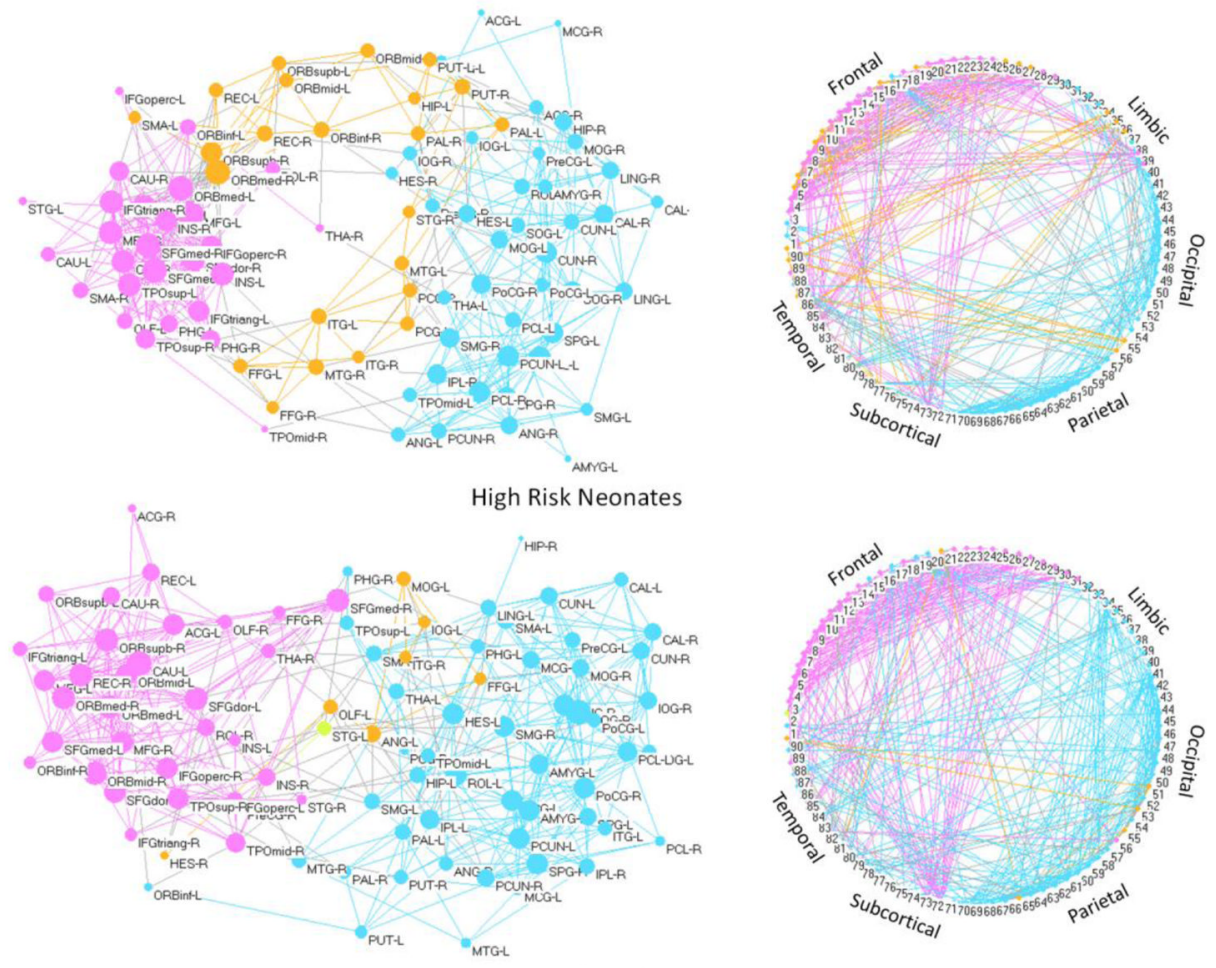

Figure 5.

Left panel shows the spring-embedded visualization of the morphological networks. Nodes are color-coded based on different modules. Intra-module edges are color-coded as the same color with the nodes. Gray edges connect inter-modules. Right panel shows circular visualization of the networks, where the nodes are arranged clockwise based on their indices, dividing them into 6 major lobes: frontal, limbic, occipital, parietal, subcortical, and temporal lobes. Note that all subjects in each population are used to construct the network, and the network sparsity is set as $12 \%$. 

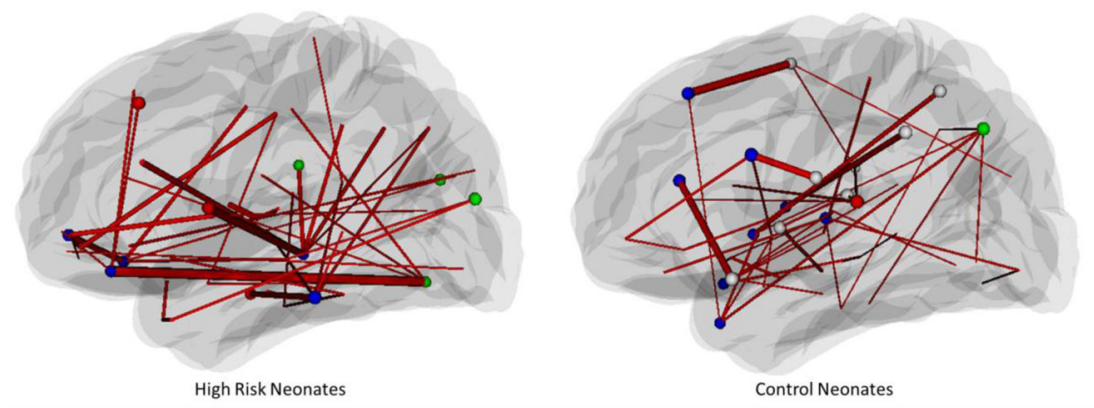

Figure 6.

Hub nodes and edges for morphological networks of high-risk neonates (top) and control neonates (bottom). Hub nodes are denoted as spheres and the colors represent different modules. Thicker edges indicate larger betweenness values, implying that they are traversed more frequently. Hub nodes are listed in Table 2, and the top 5 hub edges are listed in Table 3. Note that the two ends of the hub edges are not necessarily hub nodes, and non-hub nodes are not shown in the figure. 

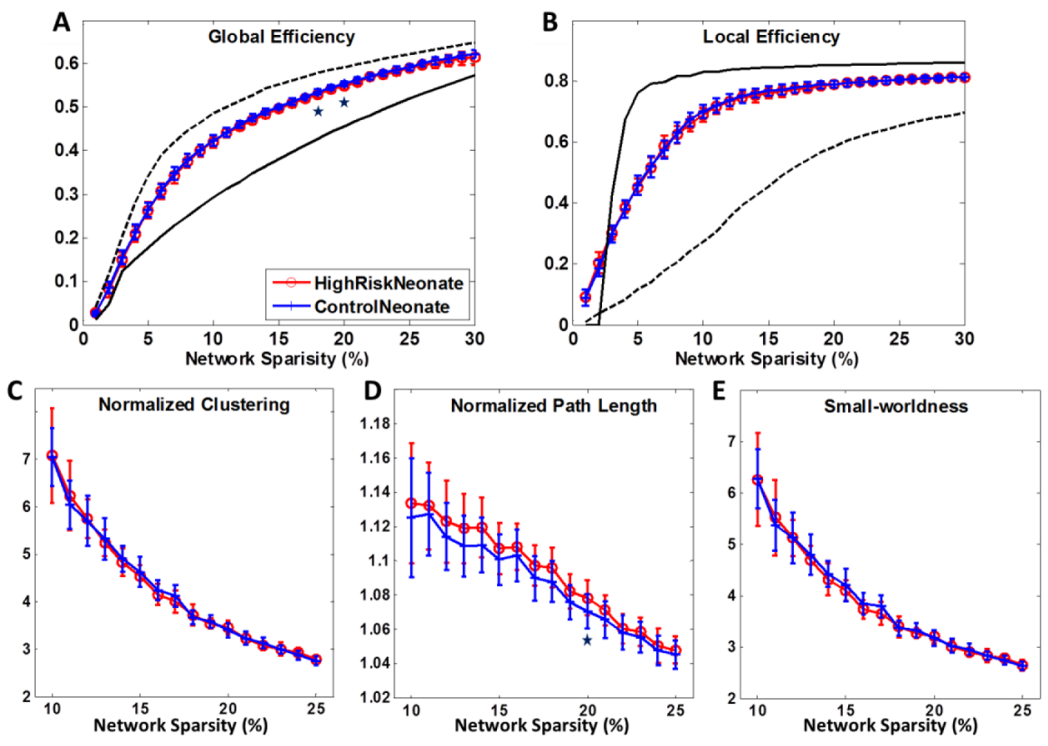

Figure 7.

Network properties of (A) global efficiency, (B) local efficiency, (C) normalized clustering, (D) normalized path length, and (E) small-worldness as functions of network sparsity for white matter networks of high-risk neonates (red) and control neonates (blue). Mean and standard deviation of network properties are illustrated at each sparsity level. Few differences were found significant ( $\mathrm{p}<0.05$ by permutation test, marked with a star) between the two populations within sparsity of $10-25 \%$. 

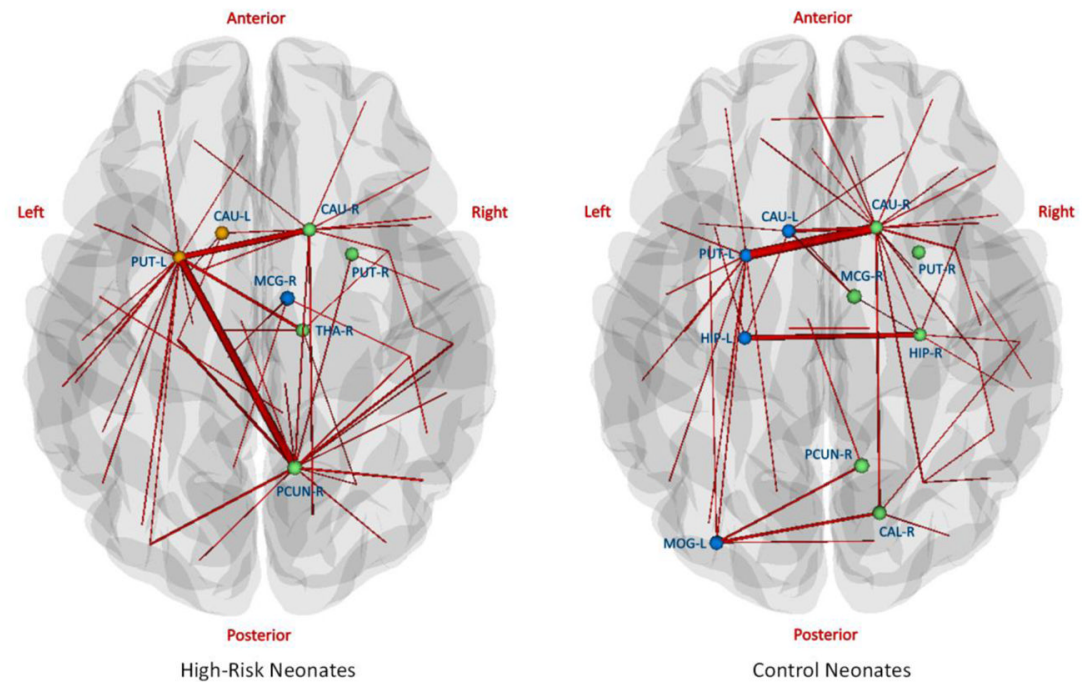

Figure 8.

Hub nodes and edges for white matter networks of high-risk neonates (left) and control neonates (right). Hub nodes are denoted as spheres and the colors represent different modules. Thicker edges indicate larger betweenness values, implying that they are traversed more frequently. Note that the two ends of the hub edges are not necessarily hub nodes, and non-hub nodes are not shown in the figure. 



Figure 9.

Visualization of connections with significantly lower number of fibers $(p<0.05$, FDR corrected) in high-risk neonates, compared with control neonates. The left and right panels show the axial and sagittal views, respectively. None of connections have significantly higher number of fibers in the high-risk neonates. 
Table 1

Demographic characteristics and prenatal and perinatal conditions of the neonates at high genetic risk for schizophrenia and the control neonates.

\begin{tabular}{|c|c|c|c|c|}
\hline \multirow[t]{2}{*}{ Characteristic, Prenatal and Perinatal Conditions $a$} & \multicolumn{2}{|c|}{ High-Risk Neonates } & \multicolumn{2}{|c|}{ Control Neonates } \\
\hline & $\mathbf{N}$ & $\%$ & $\mathbf{N}$ & $\%$ \\
\hline \multicolumn{5}{|l|}{ Gender } \\
\hline Male & 12 & 46.2 & 12 & 46.2 \\
\hline Female & 14 & 53.8 & 14 & 53.8 \\
\hline \multicolumn{5}{|l|}{ Ethnicity } \\
\hline Caucasian & 17 & 65.4 & 12 & 46.2 \\
\hline African American & 9 & 34.6 & 13 & 50.0 \\
\hline Other & 0 & 0.0 & 1 & 3.8 \\
\hline \multicolumn{5}{|l|}{ Medication Exposure } \\
\hline Antipsychotic ${ }^{*}$ & 0 & 0.0 & 23 & 88.5 \\
\hline Antidepressant ${ }^{*}$ & 0 & 0.0 & 8 & 30.8 \\
\hline Lithium & 0 & 0.0 & 2 & 7.7 \\
\hline Other Medications & 16 & 61.5 & 18 & 69.2 \\
\hline \multicolumn{5}{|l|}{ Substance Exposure } \\
\hline Tobacco Smoking * & 1 & 3.8 & 12 & 46.2 \\
\hline Alcohol & 0 & 0.0 & 3 & 11.5 \\
\hline Marijuana & 0 & 0.0 & 4 & 15.4 \\
\hline Caesarean Section & 7 & 26.9 & 12 & 46.2 \\
\hline Premature Birth (<37 weeks) & 8 & 30.8 & 7 & 26.9 \\
\hline \multirow[t]{2}{*}{ Stay in Neonatal Intensive Care Unit } & 2 & 7.7 & 6 & 23.1 \\
\hline & Mean & SD & Mean & $\mathrm{SD}$ \\
\hline Maternal Age (years) & 27.9 & 4.5 & 29.0 & 4.0 \\
\hline Maternal Education (years) ${ }^{*}$ & 14.2 & 2.7 & 10.0 & 3.6 \\
\hline \multicolumn{5}{|l|}{ Neonatal Age (weeks since mother's last menstrual period) } \\
\hline At birth & 38.1 & 3.1 & 38.5 & 2.3 \\
\hline At MRI & 43.2 & 3.3 & 42.6 & 2.4 \\
\hline \multicolumn{5}{|l|}{ Apgar Score $b$} \\
\hline 1 minute & 7.3 & 2.1 & 8.0 & 1.2 \\
\hline 5 minutes $*$ & 8.5 & 1.1 & 9.0 & 0.3 \\
\hline
\end{tabular}




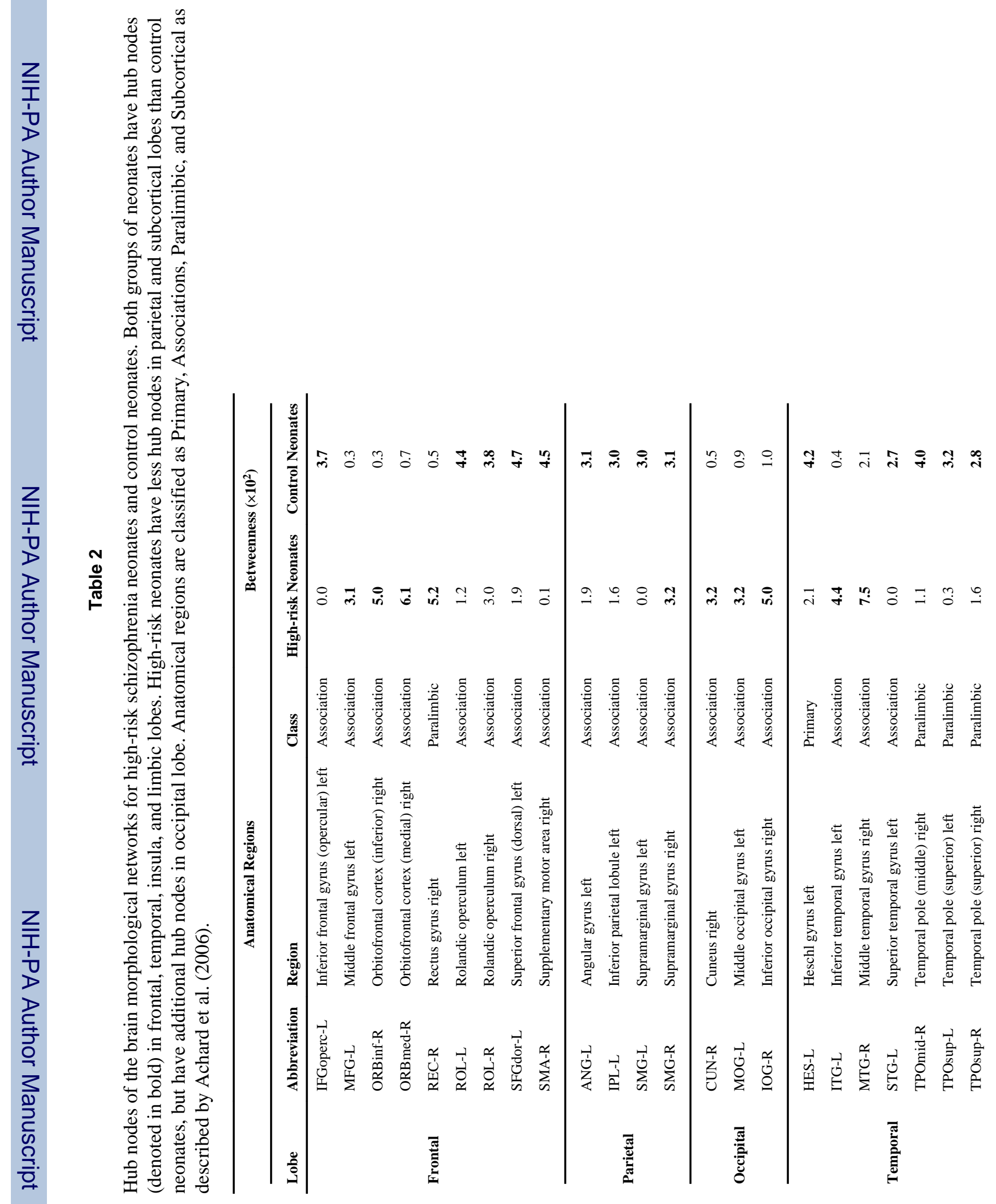




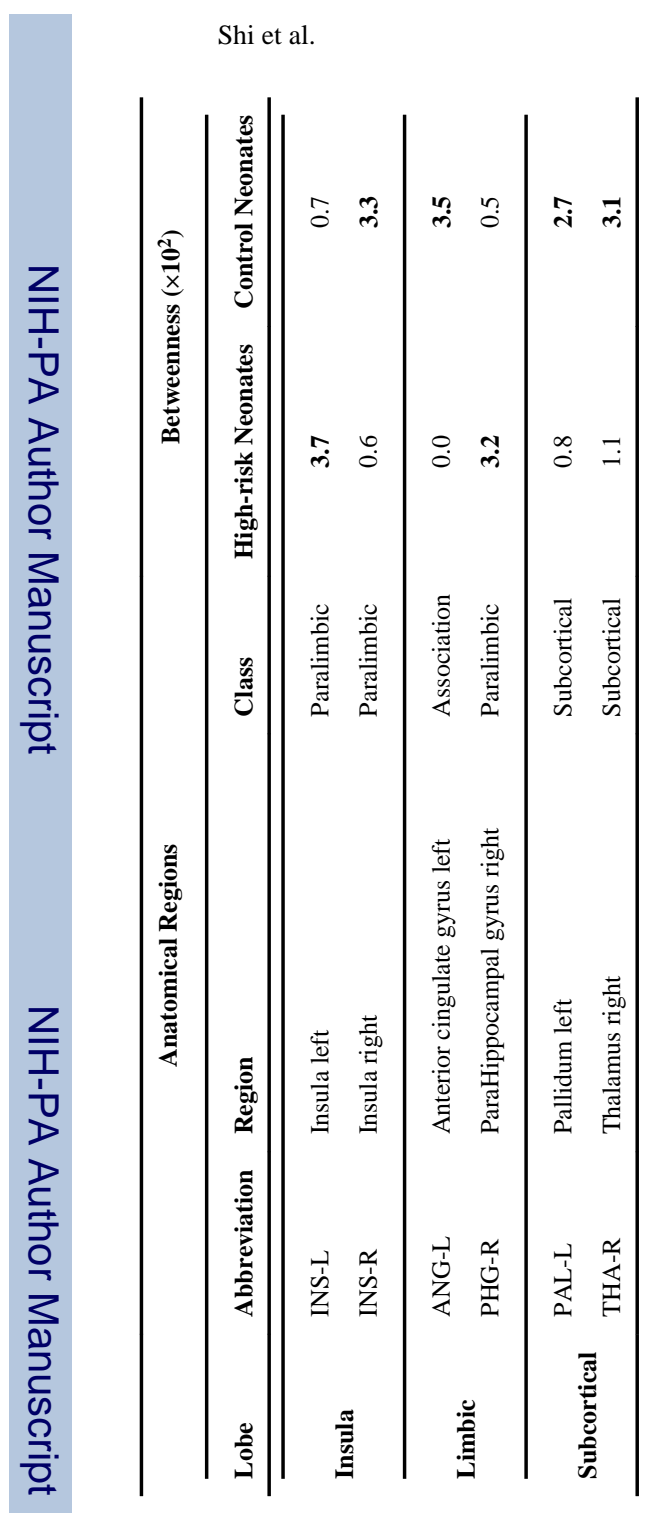

Page 27 


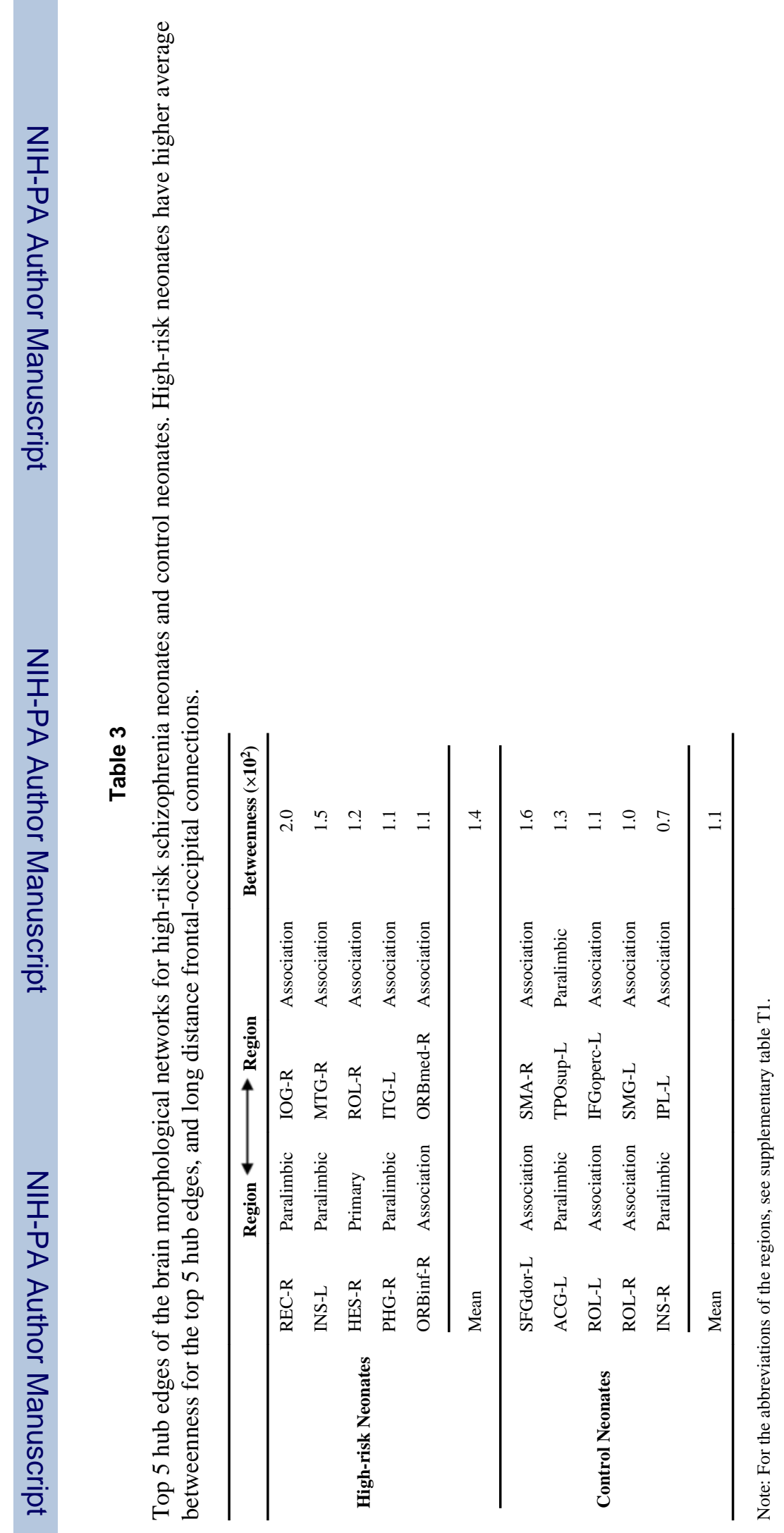

